\title{
Selecting surgical candidates with locally advanced pancreatic cancer: a review for modern pancreatology
}

\author{
Y. H. Andrew Wu ${ }^{1,2 \#}$, Atsushi Oba ${ }^{1,3 \#}$, Ronggui Lin ${ }^{1,4}$, Shuichi Watanabe ${ }^{1,5}$, Cheryl Meguid ${ }^{1}$, \\ Richard D. Schulick ${ }^{1,6}$, Marco Del Chiaro ${ }^{1,6}$
}

${ }^{1}$ Division of Surgical Oncology, Department of Surgery, University of Colorado School of Medicine, Anschutz Medical Campus, Aurora, CO, USA; ${ }^{2}$ Department of Surgery, Johns Hopkins University School of Medicine, Baltimore, MD, USA; ${ }^{3}$ Department of Hepatobiliary and Pancreatic Surgery, Cancer Institute Hospital, Japanese Foundation for Cancer Research, Tokyo, Japan; ${ }^{4}$ Department of General Surgery, Fujian Medical University Union Hospital, Fuzhou, China; ${ }^{5}$ Department of Hepato-Biliary-Pancreatic Surgery, Graduate School of Medicine, Tokyo Medical and Dental University, Tokyo, Japan; ${ }^{6}$ University of Colorado Cancer Center, Aurora, CO, USA

Contributions: (I) Conception and design: All authors; (II) Administrative support: None; (III) Provision of study material or patients: None; (IV) Collection and assembly of data: YHA Wu, A Oba; (V) Data analysis and interpretation: YHA Wu, A Oba, R Lin, S Watanabe; (VI) Manuscript writing: All authors; (VII) Final approval of manuscript: All authors.

"These authors contributed equally to this work.

Correspondence to: Marco Del Chiaro, MD, PhD, FACS. Division Chief Surgical Oncology, Director of the Hepato-Biliary-Pancreatic Program, Director of the NPF designated National Center of Excellence for Pancreatic Cancer, Department of Surgery, University of Colorado Anschutz Medical Campus, Aurora, CO, USA. Email: marco.delchiaro@cuanschutz.edu.

\begin{abstract}
Pancreatic cancer (PC) is likely to become the second leading cause of malignancy-associated mortality within the next 10 years and surgery remains the best hope for cure. The introduction of effective neoadjuvant treatment (NAT) has increased the resection rate of PC in the era of contemporary pancreatology. This review summarizes the surgical selection criteria for locally advanced PC (LAPC), by focusing on the commonly used predictors for resectability and better overall survival outcome. Based on the currently available evidence, the role of change in carbohydrate antigen 19-9 (CA 19-9) and patient's tumor response to NAT are critical in surgical candidacy selection. Although, consensus on surgical candidacy selection for LAPC still needs to be made, several data have shown that surgery provides the most optimistic chance of cure for PC. Surgery is, therefore, recommended whenever the benefits of pancreatectomy outweigh surgical complications, and the chance of local or distant metastases in the postoperative setting is low. This review also provided our insight for and experience in selecting surgical candidates by focusing on optimizing the overall survival of LAPC patients. Nevertheless, a collaborative approach to formulating standardized criteria for surgical candidate selection and treatment guidelines for LAPC is a common goal that pancreatologists worldwide should focus on.
\end{abstract}

Keywords: Locally advanced pancreatic cancer (LAPC); borderline resectable pancreatic cancer (borderline resectable PC); neoadjuvant treatment (NAT); resectability; carbohydrate antigen 19-9 (CA 19-9)

Submitted Oct 04, 2020. Accepted for publication May 14, 2021.

doi: 10.21037/jgo-21-119

View this article at: http://dx.doi.org/10.21037/jgo-21-119

\section{Introduction}

Management of pancreatic cancer (PC) remains a formidable challenge. Despite our constant effort in investigating the biology of PC and improving our therapeutic approaches (1), PC is very likely to become the second main cause of malignancy-associated mortality within the next 10 years (2). Since the identification of population at risk for early cancer detection and prevention remains difficult, only approximately $20 \%$ of the patients are candidates for pancreatectomy at the moment of 
diagnosis (3). The difficulty in the management of PC lies in the selection of surgical candidates from the population with borderline resectable or locally advanced PC (LAPC). There is no consensus on the selection of surgical candidacy for LAPC, despite the general effort in maximizing survival with available treatment methods. The time point to perform surgery is also debatable. In general, surgeons need to operate when recurrence chances are low.

According to National Comprehensive Cancer Network (NCCN) guideline on pancreatic adenocarcinoma (version $1,2020)$, LAPC is defined as a tumor with $>180^{\circ}$ contact with celiac and/or superior mesenteric artery, invasion/ occlusion that results in unreconstructable portal and superior mesenteric vein, or invasion of celiac artery with aortic involvement (4). Thanks to the introduction of more effective chemotherapy, including FOLFIRINOX and gemcitabine-nab-paclitaxel $(\mathrm{GnP})$, within the last 10 years, patients with LAPC have higher chances of being operable after receiving neoadjuvant treatment (NAT) $(5,6)$. Considering that conventional cross-sectional imaging cannot discriminate the difference between cancer tissue and post-neoadjuvant scar tissue, the absence of radiological evidence of tumor progression after NAT should be regarded as a possible effective treatment response $(7,8)$. Recently published series have shown improvements in the long-term survival of patients who underwent aggressive surgery for LAPC after NAT. It is evident that NAT has revitalized the role of vascular resections in pancreatic surgery, and has made new and more innovative techniques like artery divestment more achievable $(9,10)$.

Studies have presented factors that predict resectability in cases of LAPC. Pre- and post-neoadjuvant carbohydrate antigen 19-9 (CA 19-9) serum levels have often been compared for their ability to predict chances of successful resection and overall survival. Similarly, tumor responses to the neoadjuvant chemotherapy based on cross-sectional imaging findings, following the Response Evaluation Criteria in Solid Tumors (RECIST) (11), have often been described and debated as an important reference for deciding surgical candidacy. It is important to emphasize that conventional radiology cannot accurately determine the extent of true tumor involvement after NAT (8).

This review paper describes existing approaches in selecting LAPC patients for tumor resection based on the currently available evidence. In conjunction with the summary of recent publications, our group also provided our insight for and experience of selecting surgical candidates focusing on optimizing the overall survival of
LAPC patients. A collaborative approach on formulating a standardized criterion for surgical candidate selection and treatment guidelines for LAPC is a common goal that pancreatologists worldwide should focus on.

\section{Role of NAT in the selection of surgical candidates with LAPC}

Surgical intervention is the only potentially curative modality of treatment for PC. The role of NAT in $\mathrm{PC}$ is indispensable in achieving resectability in the initially inoperable patients. In the era prior to the introduction of FOLFIRINOX and GnP, surgery after NAT with gemcitabine with or without radiotherapy (RT) of LAPC was extremely rare (12). Within the last decade, the introduction of FOLFIRINOX and GnP has played a significant role in improving the overall survival of PC patients $(5,6,13)$, as LAPC patients would more likely become operable after undergoing more effective chemotherapy treatment (14-19). Therefore, FOLFIRINOX and GnP are currently the preferred firstline regimens in the neoadjuvant setting for patients with LAPC. Considering that PC is almost always a systemic disease at the moment of diagnosis and that $\mathrm{PC}$ recurrence is generally represented as distant metastasis or local recurrence in $23.7 \%$ of cases (20), the primary objective of NAT in LAPC should not be focused on tumor size reduction for achieving easier resection (21). Instead, the systemic treatment approach should focus on stratifying patients by tumors of different levels of biological aggressiveness according to response to NAT. Several attempts have been made to stratify patient prognosis based on the overall characteristics of a tumor instead of purely on anatomic features (22-24). With the improvement of oncologic therapy, studies have shown that arterial resections in well-selected cases can achieve similar longterm overall survival in experienced centers $(9,25,26)$. The shift in this concept is critical as several reports have indicated that surgical resection after NAT for LAPC, even for metastatic PC, can give a significant increase in survival rate and even a chance for cure $(16,18,19)$. Oba et al. have shown that administration of NAT and CA 19-9 levels are two of the eight prognostic factors of overall survival in the preoperative setting (21). However, there is no consensus with regards to how long NAT needs to be administered before proceeding to surgical treatment (Table 1). Some have indicated that $\geq 8$ months of NAT is associated with better prognosis and resectability $(17,19)$. Conversely, some 


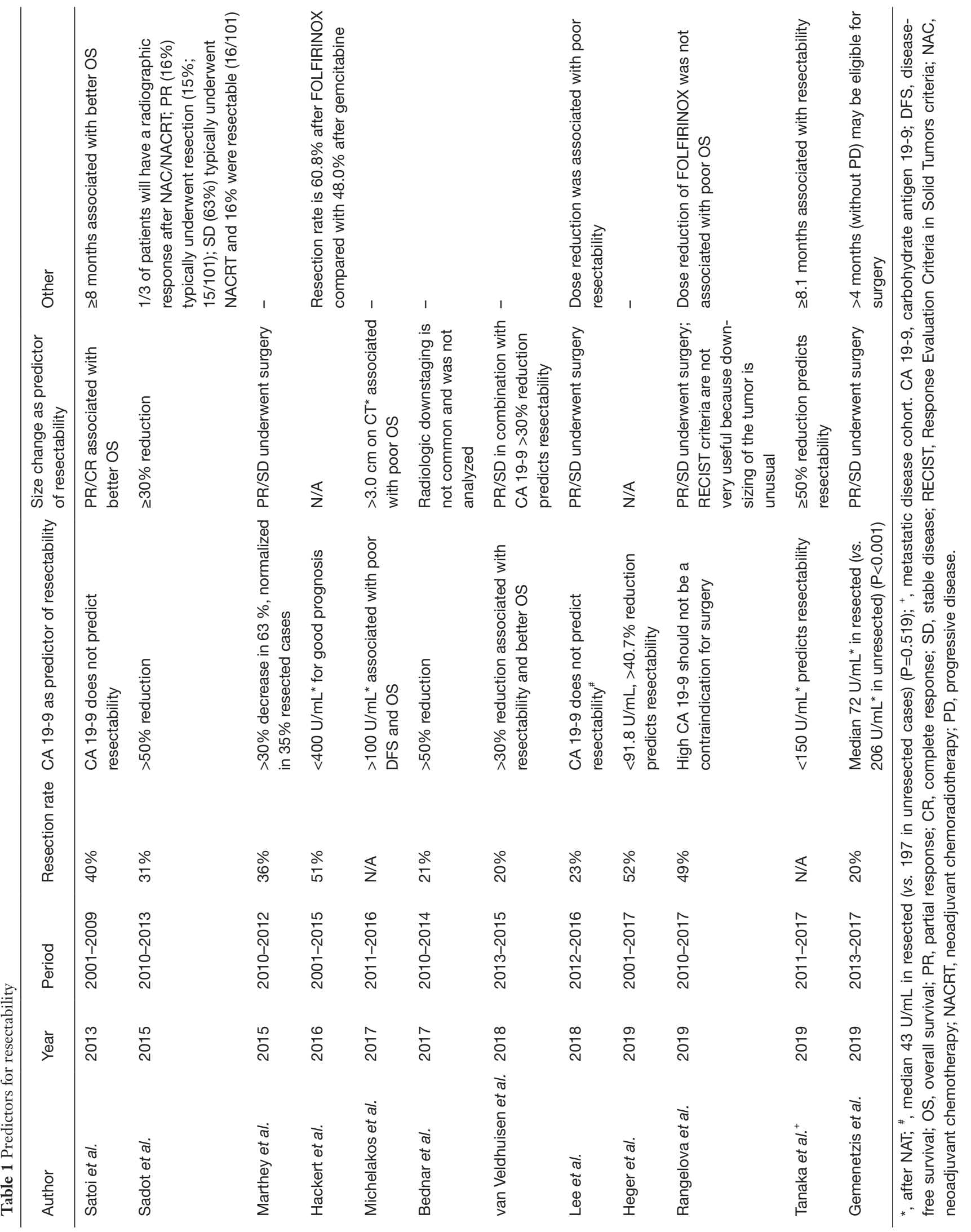


have argued that with radiological evidence showing no signs of disease progression, surgery may be warranted even after just $\geq 4$ months of NAT (16). Although some studies have indicated that dose reduction of NAT is associated with poor resectability (27), Rangelova and colleagues have stated that this association does not apply to FOLFIRINOX (15). In fact, in the setting of successful surgical resection, dose reduction of mono-chemotherapy or other combination therapy did not seem to have a negative impact on survival (15). With resection rate reported to be as high as $51.9-61 \%$ after NAT $(14,28)$, these results highlighted the potential synergistic effect of surgery and NAT in the treatment of LAPC.

Similar to LAPC, borderline resectable PC has higher R0 resection rate ( $73 \%$ vs. $48 \% ; \mathrm{P}=0.004), 3$-year overall survival (31.9 vs. 18.1 months; $\mathrm{P}=0.014$ ) after undergoing NAT with GnP compared to conventional upfront surgery (29). Similar trends were witnessed in an RCT comparing neoadjuvant chemoradiotherapy (NACRT) with GnP and upfront surgery: both the 2-year survival rate $(40.7 \% \mathrm{vs}$. $26.1 \%$ ) and median survival (21 vs. 12 months) were in favor of NACRT (30). A phase 2 clinical trial indicated that FOLFIRINOX in the neoadjuvant setting allows patients to have a higher R0 resection rate and ultimately higher median progression-free and overall survival (31).

$\mathrm{RT}$ has been commonly incorporated in the multidisciplinary treatment of PDAC, especially in the United States (21). Several publications have compared the effects of FOLFIRINOX with or without RT. When FOLFIRINOX is given with RT, the resection rate for LAPC is $13-44 \%$ (32-36); if FOLFIRINOX is given without $\mathrm{RT}$, the resection rate is $51-100 \%$ $(14,15,37-39)$. However, it is important to keep in mind these data are derived from retrospective studies and bias could exist as patients who undergo RT could generally have more advanced tumors. A large retrospective study has indicated that there is no difference in survival in NAT with or without RT (40). It is also important to note that complications of RT including radiation-induced arteritis could hinder the execution of artery resection and reconstruction. However, when stereotactic body radiation therapy (SBRT) or intensity-modulated radiation therapy (IMRT) is performed in an experienced center, the 90-day mortality rate could be as low as $0 \%$ (41). Additional prospective studies need to be performed to verify the effectiveness of RT with chemotherapy.

\section{CA 19-9 surgical candidacy selection}

Consensus with regards to the indication of surgical resection of LAPC after NAT is still lacking. Currently available studies the focuses on using post-NAT factors as predictors of tumor are all of retrospective nature (Table 1). The common candidates proposed as potential predictors of resectability are change in CA 19-9 and change in tumor size on cross-sectional imaging after undergoing NAT. The predictive role of these factors is, however, very debatable. Studies have indicated that the significant change in CA 19-9 after NAT $(35,42-44)$ or a specific CA 19-9 serum level cutoff $(14,19,28)$ predicts resectability or good prognosis. Published data have suggested that change in CA 19-9 could reflect the efficacy of NAT (45-47) and, therefore, LAPC with reduced CA 19-9 post-NAT could be managed surgically if survival benefit is carefully weighed against the perioperative morbidity of pancreatectomy and vascular resection/reconstruction. Heger et al. described that absolute serum CA $19-9$ of $<91.8 \mathrm{U} / \mathrm{mL}$ or post-NAT/ pre-NAT CA 19-9 ratio of $<0.407$ after FOLFIRINOX is associated with resectability and better prognosis in LAPC (28). It is important to note that in the same study, the same dynamic variables CA 19-9 ratio was not associated with resectability or better prognosis when patients were given gemcitabine alone. This highlights the importance of FOLFIRINOX and GnP in modern pancreatology $(5,6)$. Similarly, Tanaka et al. and Hackert et al. have described that CA 19-9 serum levels of $<150$ and $<400 \mathrm{U} / \mathrm{mL}$ can predict resectability or overall survival in LAPC and even in metastatic PCs that underwent metastasectomy $(14,19,48)$. Preoperative reduction in CA 19-9 has also been reported to be associated with higher rates of resectability and R0 resection in PC $(48,49)$. On the other hand, several studies have indicated the inability of factors associated with CA $19-9$ to predict resectability $(17,18,27)$. Nevertheless, in a retrospective cohort of 233 LAPC cases, surgical resection was shown to have a positive impact on survival for all CA 19-9 serum level values, despite the fact that higher CA 19-9 is associated with a worse prognosis (15). The results of this study advocate the idea that high CA 19-9 serum level should perhaps not be an absolute contraindication for resection. It is also important to note that there are around $5-10 \%$ of the population who are Lewis antigennegative and are CA 19-9 none or low secretors. PC in this subset of populations is usually very aggressive and has 
high proliferative and migratory characteristics (50). Luo et al. have shown that carcinoembryonic antigen (CEA) and CA-125 are associated with tumor metastasis and therapeutic response and are the two most reliable known biomarkers for Lewis antigen-negative PC with sensitivity (and specificity) of $63.8 \%$ (98\%) and $51.1 \%(93.8 \%$ ), respectively (51).

\section{Radiological response to NAT for surgical candidacy selection}

Aside from the utilizing serum level of CA 19-9, observing the tumor response to NAT could be a viable reference for resectability, as biologically less aggressive PC would respond more to mainstream chemotherapy. RECIST is a common reference used to assess the change in tumor burden of target lesions throughout the duration of systemic treatment of solid cancer (11). It is a common reference that pancreatologists utilize to objectively evaluate the response of PC to systemic treatment. Complete response (CR) or partial response (PR) to NAT is generally associated with a better prognosis (17). Although, cross-sectional imaging may be helpful to exclude patients with definite cancer progression from pancreatectomy, it has low accuracy in determining the viability of tumor post-NAT as existing radiologic imaging modalities cannot distinguish benign fibrosis from viable malignant tissue (8). This demonstrates that previously utilized criteria for determining resectability by cross-sectional imaging are perhaps inappropriate in patients who receive modern chemotherapy, such as FOLFIRINOX and GnP. Gemenetzis et al. have shown that of the 84 patients who underwent resection due to no signs of local disease progression or metastases, $77 \%$ had pathologically proven response to NAT (16). Therefore, all patients without radiologic evidence of local or systemic disease progression should be considered for surgical exploration. If evaluations rule out the possibility of R2/R1 resection or other contraindications for surgery, pancreatectomy with or without vessel resection should be considered.

\section{LAPC resection and outcomes}

Although radiologic downstaging is unusual and is reported to be approximately $28 \%$ (52), the $\mathrm{R} 0$ resection rate in selected LAPC patients after NAT has been reported to be $>90 \%(8,52)$. Studies have correlated surgical resection of the primary tumor with significantly improved overall survival in LAPC patients, emphasizing that median survival of resected LAPC could range from 21 to 37.9 months and is significantly better than those of unresected LAPC $(\mathrm{P}<0.001)(14-17)$. Moreover, according to the newly developed nomogram that was recently published by our group, the LAPC-equivalent clinical T4 stage did not show a significantly worse prognosis in the resected cohort (23). Our work suggested that in the setting of undergoing modern chemotherapeutic agents, the role of local anatomic features of the tumor (including arterial involvement) has less importance than the biology of the disease and its response to chemotherapy (23).

Although radical surgery after NAT is expected to prolong survival, studies have shown that a $30 \%$ recurrence rate can be witnessed within 6 months postoperatively (53). Groot et al. reported that $81.8 \%$ of the 231 patients who underwent resection after NAT experienced recurrence (72.5\% of which are distant metastasis) (20). The same cohort had a median postoperative recurrence-free period and median post-recurrence survival duration of 9.8 months and 8.4 months, respectively (20). Therefore, decisions for resection after NAT should be thoroughly evaluated in multi-disciplinary meetings and personalized treatment methodology that focuses on optimal timing between initial treatment and resection, accurate method of evaluating tumor remission, and patient's post-treatment quality of life should be assessed.

Due to improved surgical management, pancreatectomy has low 90-day mortality. In the modern era, this value can be as low as $2-4 \%$ when complex arterial resection is performed in an experienced center $(52,54)$. These mortalities are caused by vascular thrombosis, liver failure, pancreatic fistulas, and sepsis-related multi-organ failure (26). These good results should be evaluated carefully. The large majority of studies on LAPC involve a small and very well selected group of patients, therefore there is a presence of selection bias. In a recent and large series published by one of the most experienced groups in the treatment of LAPC, the overall mortality rate was $8.8 \%$, with a significant decrease to $4.8 \%$ in recent years (25).

\section{Conclusions}

Currently available NAT has significantly increased the survival in PC and have, therefore, increased the rate of resectability for patients affected by LAPC. However, a 
consensus regarding the definition of resectability of PC does not exist. The majority of the current criteria is mostly anatomic-based. This approach has limitations, since we cannot accurately describe the biological response of the tumor to NAT through radiological imaging in many cases. Similar to the development of treatment for other cancers (55-57), the effectiveness of NAT for PC will improve and the surgical indications for PC will be extended so that more patients could benefit from surgical resection. Since the introduction of highly effective NAT for PC occurred only within the last decade, the majority of the available research data related to this topic are mostly of retrospective nature and, thus, have limitations related to the study type.

Future prospective randomized studies should be performed in order to investigate the algorithms for surgical candidacy selection, the adequate treatment duration of NAT, and the predictors for chemotherapy response. Future research should also focus more on the biology of PC, as anatomy-based treatment has limitations. With a better understanding of tumor biology and the emergence of more effective NAT, we should soon expect a refined definition of resectability that is more prognosis-based and is more independent of tumor anatomic involvement (23).

\section{Acknowledgments}

Funding: None.

\section{Footnote}

Provenance and Peer Review: This article was commissioned by the Guest Editor (Elena Rangelova) for the series "Surgery for Locally Advanced Pancreatic Cancer" published in fournal of Gastrointestinal Oncology. The article has undergone external peer review.

Conflicts of Interest: All authors have completed the ICMJE uniform disclosure form (available at http://dx.doi. org/10.21037/10.21037/jgo-21-119). The series "Surgery for Locally Advanced Pancreatic Cancer" was commissioned by the editorial office without any funding or sponsorship. Dr. MDC is a co-principal investigator on a Boston Scientific study investigating the role of intraoperative pancreatoscopy in IPMN patients. Dr. MDC has also been awarded an industry grant from Haemonetics Inc. to conduct a multicenter study that evaluates the prognostic implications and changes of thromboelastographic (TEG) in pancreas cancer patients. The authors have no other conflicts of interest to declare.

Ethical Statement: The authors are accountable for all aspects of the work in ensuring that questions related to the accuracy or integrity of any part of the work are appropriately investigated and resolved.

Open Access Statement: This is an Open Access article distributed in accordance with the Creative Commons Attribution-NonCommercial-NoDerivs 4.0 International License (CC BY-NC-ND 4.0), which permits the noncommercial replication and distribution of the article with the strict proviso that no changes or edits are made and the original work is properly cited (including links to both the formal publication through the relevant DOI and the license). See: https://creativecommons.org/licenses/by-nc-nd/4.0/.

\section{References}

1. Neoptolemos JP, Kleeff J, Michl P, et al. Therapeutic developments in pancreatic cancer: current and future perspectives. Nat Rev Gastroenterol Hepatol 2018;15:333-48.

2. Rahib L, Smith BD, Aizenberg R, et al. Projecting cancer incidence and deaths to 2030: the unexpected burden of thyroid, liver, and pancreas cancers in the United States. Cancer Res 2014;74:2913-21.

3. Huang L, Jansen L, Balavarca Y, et al. Resection of pancreatic cancer in Europe and USA: an international large-scale study highlighting large variations. Gut 2019;68:130-9.

4. National Comprehensive Cancer Network. Pancreatic adenocarcinoma (version 1. 2020). Available online: https://www.nccn.org/professionals/physician_gls/pdf/ pancreatic_blocks.pdf (Accessed November 26, 2019).

5. Conroy T, Desseigne F, Ychou M, et al. FOLFIRINOX versus gemcitabine for metastatic pancreatic cancer. $\mathrm{N}$ Engl J Med 2011;364:1817-25.

6. Von Hoff DD, Ervin T, Arena FP, et al. Increased survival in pancreatic cancer with nab-paclitaxel plus gemcitabine. N Engl J Med 2013;369:1691-703.

7. Oba A, Bao QR, Barnett CC, et al. Vascular resections for pancreatic ductal adenocarcinoma: vascular resections for PDAC. Scand J Surg 2020;109:18-28.

8. Ferrone CR, Marchegiani G, Hong TS, et al. Radiological and surgical implications of neoadjuvant treatment with FOLFIRINOX for locally advanced and borderline resectable pancreatic cancer. Ann Surg 2015;261:12-7. 
9. Del Chiaro M, Rangelova E, Halimi A, et al.

Pancreatectomy with arterial resection is superior to palliation in patients with borderline resectable or locally advanced pancreatic cancer. HPB (Oxford) 2019;21:219-25.

10. Del Chiaro M, Schulick RD. Commentary on: Divestment or skeletonization of the SMA or the hepatic artery for locally advanced pancreatic ductal cancer after neoadjuvant therapy. Surgery 2021;169:1039-40.

11. Eisenhauer EA, Therasse P, Bogaerts J, et al. New response evaluation criteria in solid tumours: revised RECIST guideline (version 1.1). Eur J Cancer 2009;45:228-47.

12. Hammel P, Huguet F, van Laethem JL, et al. Effect of chemoradiotherapy vs chemotherapy on survival in patients with locally advanced pancreatic cancer controlled after 4 months of gemcitabine with or without erlotinib: the LAP07 randomized clinical trial. JAMA 2016;315:1844-53.

13. Conroy T, Hammel P, Hebbar M, et al. FOLFIRINOX or gemcitabine as adjuvant therapy for pancreatic cancer. $\mathrm{N}$ Engl J Med 2018;379:2395-406.

14. Hackert T, Sachsenmaier M, Hinz U, et al. Locally advanced pancreatic cancer: neoadjuvant therapy with FOLFIRINOX results in resectability in $60 \%$ of the patients. Ann Surg 2016;264:457-63.

15. Rangelova E, Wefer A, Persson S, et al. Surgery improves survival after neoadjuvant therapy for borderline and locally advanced pancreatic cancer: a single institution experience. Ann Surg 2021;273:579-86.

16. Gemenetzis G, Groot VP, Blair AB, et al. Survival in locally advanced pancreatic cancer after neoadjuvant therapy and surgical resection. Ann Surg 2019;270:340-7.

17. Satoi S, Yamaue H, Kato K, et al. Role of adjuvant surgery for patients with initially unresectable pancreatic cancer with a long-term favorable response to non-surgical anti-cancer treatments: results of a project study for pancreatic surgery by the Japanese Society of HepatoBiliary-Pancreatic Surgery. J Hepatobiliary Pancreat Sci 2013;20:590-600.

18. Michelakos T, Pergolini I, Castillo CF, et al. Predictors of resectability and survival in patients with borderline and locally advanced pancreatic cancer who underwent neoadjuvant treatment with FOLFIRINOX. Ann Surg 2019;269:733-40.

19. Tanaka M, Heckler M, Mihaljevic AL, et al. CT response of primary tumor and CA19-9 predict resectability of metastasized pancreatic cancer after FOLFIRINOX. Eur J Surg Oncol 2019;45:1453-9.
20. Groot VP, Blair AB, Gemenetzis G, et al. Recurrence after neoadjuvant therapy and resection of borderline resectable and locally advanced pancreatic cancer. Eur J Surg Oncol 2019;45:1674-83.

21. Oba A, Ho F, Bao QR, et al. Neoadjuvant treatment in pancreatic cancer. Front Oncol 2020;10:245.

22. Paniccia A, Hosokawa P, Henderson W, et al. Characteristics of 10-year survivors of pancreatic ductal adenocarcinoma. JAMA Surg 2015;150:701-10.

23. Oba A, Croce C, Hosokawa P, et al. Prognosis based definition of resectability in pancreatic cancer: a road map to new guidelines. Ann Surg 2020. [Epub ahead of print]. doi: 10.1097/SLA.0000000000003859.

24. Brennan MF, Kattan MW, Klimstra D, et al. Prognostic nomogram for patients undergoing resection for adenocarcinoma of the pancreas. Ann Surg 2004;240:293-8.

25. Loos M, Kester T, Klaiber U, et al. Arterial resection in pancreatic cancer surgery: effective after a learning curve. Ann Surg 2020. [Epub ahead of print]. doi: 10.1097/ SLA.0000000000004054.

26. Truty MJ, Colglazier JJ, Mendes BC, et al. En bloc celiac axis resection for pancreatic cancer: classification of anatomical variants based on tumor extent. J Am Coll Surg 2020;231:8-29.

27. Lee J, Lee JC, Gromski MA, et al. Clinical outcomes of FOLFIRINOX in locally advanced pancreatic cancer: a single center experience. Medicine (Baltimore) 2018;97:e13592.

28. Heger U, Sun H, Hinz U, et al. Induction chemotherapy in pancreatic cancer: CA 19-9 may predict resectability and survival. HPB (Oxford) 2020;22:224-32.

29. Inoue $Y$, Saiura A, Oba A, et al. Neoadjuvant gemcitabine and nab-paclitaxel for borderline resectable pancreatic cancers: Intention-to-treat analysis compared with upfront surgery. J Hepatobiliary Pancreat Sci 2021;28:143-55.

30. Jang JY, Han Y, Lee H, et al. Oncological benefits of neoadjuvant chemoradiation with gemcitabine versus upfront surgery in patients with borderline resectable pancreatic cancer: a prospective, randomized, open-label, multicenter phase 2/3 trial. Ann Surg 2018;268:215-22.

31. Murphy JE, Wo JY, Ryan DP, et al. Total neoadjuvant therapy with FOLFIRINOX followed by individualized chemoradiotherapy for borderline resectable pancreatic adenocarcinoma: a phase 2 clinical trial. JAMA Oncol 2018;4:963-9.

32. Blazer M, Wu C, Goldberg RM, et al. Neoadjuvant 
modified (m) FOLFIRINOX for locally advanced unresectable (LAPC) and borderline resectable (BRPC) adenocarcinoma of the pancreas. Ann Surg Oncol 2015;22:1153-9.

33. Khushman M, Dempsey N, Maldonado JC, et al. Full dose neoadjuvant FOLFIRINOX is associated with prolonged survival in patients with locally advanced pancreatic adenocarcinoma. Pancreatology 2015;15:667-73.

34. Nanda RH, El-Rayes B, Maithel SK, et al. Neoadjuvant modified FOLFIRINOX and chemoradiation therapy for locally advanced pancreatic cancer improves resectability. J Surg Oncol 2015;111:1028-34.

35. Sadot E, Doussot A, O'Reilly EM, et al. FOLFIRINOX induction therapy for stage 3 pancreatic adenocarcinoma. Ann Surg Oncol 2015;22:3512-21.

36. Suker M, Beumer BR, Sadot E, et al. FOLFIRINOX for locally advanced pancreatic cancer: a systematic review and patient-level meta-analysis. Lancet Oncol 2016;17:801-10.

37. Barenboim A, Lahat G, Geva R, et al. Neoadjuvant FOLFIRINOX for locally advanced and borderline resectable pancreatic cancer: an intention to treat analysis. Eur J Surg Oncol 2018;44:1619-23.

38. Byun Y, Han Y, Kang JS, et al. Role of surgical resection in the era of FOLFIRINOX for advanced pancreatic cancer. J Hepatobiliary Pancreat Sci 2019;26:416-25.

39. Nitsche U, Wenzel P, Siveke JT, et al. Resectability after first-line FOLFIRINOX in initially unresectable locally advanced pancreatic cancer: a single-center experience. Ann Surg Oncol 2015;22 Suppl 3:S1212-20.

40. Vela N, Davis LE, Cheng SY, et al. Economic analysis of adjuvant chemoradiotherapy compared with chemotherapy in resected pancreas cancer. Ann Surg Oncol 2019;26:4193-203.

41. Chapman BC, Gleisner A, Rigg D, et al. Perioperative outcomes and survival following neoadjuvant stereotactic body radiation therapy (SBRT) versus intensity-modulated radiation therapy (IMRT) in pancreatic adenocarcinoma. J Surg Oncol 2018;117:1073-83.

42. Marthey L, Sa-Cunha A, Blanc JF, et al. FOLFIRINOX for locally advanced pancreatic adenocarcinoma: results of an AGEO multicenter prospective observational cohort. Ann Surg Oncol 2015;22:295-301.

43. van Veldhuisen E, Vogel JA, Klompmaker S, et al. Added value of CA19-9 response in predicting resectability of locally advanced pancreatic cancer following induction chemotherapy. HPB (Oxford) 2018;20:605-11.
44. Bednar F, Zenati MS, Steve J, et al. Analysis of predictors of resection and survival in locally advanced stage III pancreatic cancer: does the nature of chemotherapy regimen influence outcomes? Ann Surg Oncol 2017;24:1406-13.

45. Aldakkak M, Christians KK, Krepline AN, et al. Pretreatment carbohydrate antigen 19-9 does not predict the response to neoadjuvant therapy in patients with localized pancreatic cancer. HPB (Oxford) 2015;17:942-52.

46. Katz MH, Fleming JB, Bhosale P, et al. Response of borderline resectable pancreatic cancer to neoadjuvant therapy is not reflected by radiographic indicators. Cancer 2012;118:5749-56.

47. Boone BA, Steve J, Zenati MS, et al. Serum CA 19-9 response to neoadjuvant therapy is associated with outcome in pancreatic adenocarcinoma. Ann Surg Oncol 2014;21:4351-8.

48. Hartwig W, Strobel O, Hinz U, et al. CA19-9 in potentially resectable pancreatic cancer: perspective to adjust surgical and perioperative therapy. Ann Surg Oncol 2013;20:2188-96.

49. Hartwig W, Hackert T, Hinz U, et al. Pancreatic cancer surgery in the new millennium: better prediction of outcome. Ann Surg 2011;254:311-9.

50. Liu C, Deng S, Jin K, et al. Lewis antigennegative pancreatic cancer: an aggressive subgroup. Int J Oncol 2020;56:900-8.

51. Luo G, Liu C, Guo M, et al. Potential biomarkers in Lewis negative patients with pancreatic cancer. Ann Surg 2017;265:800-5.

52. Truty MJ, Kendrick ML, Nagorney DM, et al. Factors predicting response, perioperative outcomes, and survival following total neoadjuvant therapy for borderline/locally advanced pancreatic cancer. Ann Surg 2021;273:341-9.

53. Satoi S, Yamamoto T, Yamaki S, et al. Surgical indication for and desirable outcomes of conversion surgery in patients with initially unresectable pancreatic ductal adenocarcinoma. Ann Gastroenterol Surg 2019;4:6-13.

54. Schmocker RK, Wright MJ, Ding D, et al. An aggressive approach to locally confined pancreatic cancer: defining surgical and oncologic outcomes unique to pancreatectomy with celiac axis resection (DP-CAR). Ann Surg Oncol 2021;28:3125-34.

55. Watanabe M, Otake R, Kozuki R, et al. Recent progress in multidisciplinary treatment for patients with esophageal cancer. Surg Today 2020;50:12-20. 
56. Kim HS, Kim NK. Challenges and shifting treatment strategies in the surgical treatment of locally advanced rectal cancer. Ann Gastroenterol Surg 2020;4:379-85.

Cite this article as: $\mathrm{Wu}$ YHA, Oba $\mathrm{A}$, Lin $\mathrm{R}$, Watanabe S, Meguid C, Schulick RD, Del Chiaro M. Selecting surgical candidates with locally advanced pancreatic cancer: a review for modern pancreatology. J Gastrointest Oncol 2021;12(5):2475-2483. doi: 10.21037/jgo-21-119
57. Osterman E, Hammarström K, Imam I, et al. Recurrence risk after radical colorectal cancer surgery-less than before, but how high is it? Cancers (Basel) 2020;12:3308. 